Isaac Almendros, Hani N. Alsafadi, Deniz Bölükbas,

Authors listed alphabetically except for the last author. For a list of their affiliations,

please see the Acknowledgements section.

\title{
Early Career Members at the ERS Lung Science Conference: cell-matrix interactions in lung disease and regeneration
}

Cite as: Almendros I, Alsafadi HN, Bölükbas D, et al. Early Career Members at the ERS Lung Science Conference: cell-matrix interactions in lung disease and regeneration. Breathe 2018; 14: e78-e83.

\section{Early career forum}

The 16th ERS Lung Science Conference (LSC) took place on March 8-11, 2018, in Estoril, Portugal, with around 200 delegates from all over the world. This year's topic was "Cell-matrix interactions in lung disease and regeneration" and involved excellent presentations by leading experts in the field covering everything from exploratory studies on how the matrix functions, matrix remodelling and biomarkers in disease, to more technical knowledge described in the field of lung bioengineering. As in previous years, the Saturday afternoon was reserved for a programme dedicated to early career delegates, which this year focussed on "Maximising your publication output". In this article, we summarise the Early Career Member highlights of this year's LSC.

\section{Matrix remodelling in lung disease}

The topic of the LSC opening session was the role of matrix remodelling in the pathogenesis of lung diseases. Four fruitful presentations, as well as the discussion guided by session chairs Pieter Hiemstra (Leiden, The Netherlands) and Argyris Tzouvelekis (Athens, Greece), further corroborated the evidence that matrix remodelling plays a cardinal role in chronic lung disease pathogenesis. In particular, Tracy Hussell (Manchester, UK) provided an overview of the role of matrix dysfunction in chronic obstructive pulmonary disease (COPD). Hyaluronan was found to be increased during exacerbations in patients with COPD and interestingly, further increased after influenza infection [1]. In this direction, experimental data showed that hyaluronidase treatment restored lung function and removed proteins bound to hyaluronan. Given that hyaluronan increase also affected the let- 7 microRNA (miRNA) family, further investigation of mitophagy and autophagy might hold promise. In addition to hyaluronan, prolonged changes in the basement membrane and extracellular matrix (ECM) following acute and chronic lung disease were described, and importantly, these were disease severity dependent. Furthermore, matrix impaired macrophage responses and 
upregulated several anti-inflammatory miRNAs. In a short oral presentation selected from abstracts, Gerald Burgstaller (Munich, Germany) presented a phenotypic high-throughput screening assay for the pharmacological inhibition of the pathological deposition of ECM [2]. This assay was applicable to every antigenic ECM protein of the matrisome and to any adherent cell type. A typical paradigm demonstrated was the reduction of expression of collagen by ethyl-3,4-dihydroxybenzoate. Finally, the potential of this technique to assess drug repurposing and deconvolution techniques was discussed. Martin Kolb (Hamilton, Canada) summarised his presentation for the role of ECM in pulmonary fibrosis with three key points. The first key point was alveolar epithelial injury and subsequent alveolar collapse. In this context, the role of biomarker mucin 5B was highlighted. The second key point and the key to progression was the proximity of myofibroblasts to fibrotic matrix, which influences their behaviour. The third key point was that breathing itself could amplify fibrosis due to damage occurring by regular stretching of a stiffer matrix, which is due to several different cellular pathways [3]. The winner of the best oral presentation, Marko Nikolic (Cambridge, UK), provided insights into genetically modifiable, three-dimensional organoid culture of human embryonic lung stem cells enabling, for the first time, the investigation of human lung development in vitro [4]. The role of SRY box 9 in organoid selfrenewal was emphasised. These data hopefully open new avenues for end-stage lung diseases, premature neonates and rare congenital lung conditions.

\section{Matrix-cell interactions}

The interplay between cells and matrix is often underestimated as most people study isolated in vitro systems of matrix or cells, mimicking only partly what occurs in the intact lung. Kristian Riesbeck (Malmö, Sweden) showed excellent electron microscopic images of bacterial-matrix interaction and how the bacteria attach to ECM structures such as collagen and laminin. He also showed how, sometimes, matrix molecules such as vitronectin can protect, for example, Pseudomonas aeruginosa against complement lysis. Franz Puttur (London, UK) presented, in his short oral presentation, how innate lymphoid cells (ILCS) navigate in the lung matrix, and showed amazing videos during his talk that demonstrated how collagen IV and fibronectin can support ILC2 movement. In addition, he demonstrated that collagen I was able to induce elongation of ILC2, and that blocking of the collagen crosslinking enzyme, lysyl oxidase, changed ILC2 movement by increasing their speed and travel distance. As well as attachment of bacteria to the matrix and movement of immune cells through the matrix, the session contained an oral presentation on how mutated cells unexpectedly affected the interaction with the ECM, as was shown by Giulia Maria Stella (Pavia, Italy). She also demonstrated how this may play an important role in both idiopathic pulmonary fibrosis (IPF) and metastatic growth and invasion. This was followed by a presentation by Robert Snelgrove (London, UK), who talked about the matrikines in lung inflammation, where Pro-Gly-Pro (proteolytic products of collagen) were described to function as alarmins. In the final presentation, Yuval Rinkevich (Munich, Germany) presented his work on specialised fibroblasts and their role in wound healing. He showed impressive images and data indicating different roles played by fibroblasts and their power to function as wound healing or scarinducing cells.

\section{The instructive matrix}

Saturday began with Bernhard Wehrle-Haller (Geneva, Switzerland) explaining that the interaction points between integrin and ECM proteins might be sites of signalling. This transduction of mechanical signals into chemicals signals ("mechanotransduction") is tightly regulated; one example is the movement of integrins along the cell membrane. Moreover, he explained that integrin $\alpha$ ll $\beta 3$ subunit activation is a complex process dependent on conformational changes that require integrin binding to several proteins in an allosteric way. It was also emphasised that once integrins are activated, there is a tension-controlled recruitment of signalling proteins within the intracellular space. Jae-Won Shin (Chicago, IL, USA) showed, in a short oral presentation, his work on mesenchymal stem cell (MSC)-based therapy for lung fibrosis treatment and how encapsulation of single MSCs in thin alginate microgels enhanced the retention of the cells in an animal model. Furthermore, in order to induce a proinflammatory phenotype, MSCs can be mechanical pre-activated by stiffness. In the talk entitled "When is an alveolar epithelial cell an alveolar epithelial cell?", Michael F. Beers (Philadelphia, PA, USA) pointed the main characteristics of alveolar type II (ATII) cells. ATII cells have a unique pulmonary surfactant metabolism linked to a unique lysosomal organelle with lamellar bodies full of surfactant, showing that surfactant exocytosis and endocytosis are precisely regulated. Finally, he showed that ATII cells are characterised as being lung stem cells. The session was concluded by another oral presentation by llan Azuelos (Montreal, Canada), whose work is focused on IPF research, showing how mammalian target of rapamycin (mTOR) regulates transforming growth factor (TGF)- $\beta$-induced collagen synthesis via increased glycine biosynthesis. It has been seen that fibrotic areas have enhanced glucose metabolism, which is regulated by the mTOR axis. The mTOR axis, which has been also related to glycine synthesis, is activated upon TGF- $\beta$ stimulation. Elegant results were presented in which mTOR inhibition resulted in a collagen synthesis downregulation, while this effect was reversed by adding glycine. 


\section{Young investigator session: the William MacNee Award}

Like every year at the LSC, in 2018, five young investigators who submitted the best abstracts were competing for the prestigious William MacNee Award. Jennifer Collins (Rotterdam, the Netherlands) showed how in vivo hyperoxia exposure of neonatal rats impaired the angiogenic supportive capacity and fibroblast growth factor expression in lung mesenchymal stromal cells in the context of bronchopulmonary dysplasia. Emmeline Marchal-Duval (Paris, France) shared her work on paired related homeobox 1 , a profibrotic mesenchymal transcription factor that may contribute to the development of IPF by keeping fibroblasts in a proliferative and undifferentiated state. Catharina Mueller (Lund, Sweden), who later won the award, studies the ECM of transplanted lungs in a clever combinatory approach of laser capture microdissection paired with mass spectrometry and immunohistochemistry. Based on early alterations in the ECM, she was able to identify patients likely to develop bronchiolitis obliterans syndrome and predict who will have a severe disease course. Isabelle Dupin (Bordeaux, France) demonstrated that fibrocyte-like cells are increased in distal tissue samples of COPD patients and may be involved in the lung function decline during COPD progression, showing a negative association of fibrocyte-like cell density with lung function and a positive association with bronchial wall thickness. Scott Collum (Houston, TX, USA) concluded the session with his work on alternative polyadenylation (APA), a mechanism that typically results in a shortening of the $3^{\prime}$ untranslated region of affected genes, which can lead to the removal of regulation sites and increased expression of these mRNAs. He found that APA induced by the depletion of the 25-kDa subunit of cleavage factor I contributes to the upregulation of ECM components and the development of pulmonary hypertension.

\section{Poster sessions}

Around 80 Early Career Member delegates had the chance to present their research in two poster sessions during the LSC. Several posters in the first session highlighted potent signalling pathways that contribute to the pathological activity of fibroblasts in IPF. Mimicking breathing by exerting cyclical stretch on fibroblasts was shown to release endogenous TGF- $\beta$, a process that is mediated by G-protein signalling (by G $\alpha q / 11$ ), as presented by Amanda Goodwin (Nottingham, UK). On a different note, silencing members of the A-kinase anchoring proteins demonstrated a role in mediating epithelial to mesenchymal transition that contributes to the pathogenesis of fibrosis, as presented by Martina Schmidt (Groningen, the Netherlands). Furthermore, the switch between myogenic and lipogenic fibroblast phenotype was proposed to have therapeutic benefits in IPF. Lipogenic fibroblasts are lipid droplet-containing interstitial fibroblasts that contribute to epithelial maturation and provide a niche for epithelial stemness. During fibrotic events, lipogenic fibroblasts contribute to pathogenesis by switching into a myogenic phenotype. Peroxisome proliferator-activated receptor (PPAR) $-\gamma$ signalling was found to inhibit the switch from lipogenic to myogenic phenotype and promote the differentiation of the lipogenic phenotype. Treatment with metformin, a wellknown antidiabetic drug and a PPAR- $\gamma$ agonist, on precision-cut lung slices derived from IPF patients showed amelioration of the fibrotic phenotype. This project was presented by Vahid Kheirollahi (Giessen, Germany) and was selected for a distinguished poster award. Understanding intracellular pathways and mechanisms that contribute to disease pathogenesis and ECM derangement could greatly aid in finding novel therapies that may cure chronic lung diseases. Furthermore, exciting data were presented that type 2 iodothyronine deiodinase, the enzyme that converts thyroxine to active triiodothyronine, was upregulated in the lungs of patients with IPF, particularly in alveolar epithelial cells, the metabolically active cells of the lung. In this direction, experimental data demonstrated that aerosolised thyroid hormone administration exerted antifibrotic properties in two experimental models of pulmonary fibrosis through a mechanism involving improved mitochondrial function and mitophagy. Another interesting poster further enhanced knowledge of the role of kinases and phosphatases in pulmonary fibrosis, as mitogenactivated protein kinase phosphatase- 5 blunted fibrotic responses through negative regulation of TGF- $\beta 1$-induced Smad3 signalling. Interesting data were also presented on the antifibrotic properties of azithromycin and metformin. Several mechanisms have been suggested, yet investigation of these compounds in a clinical setting remains a challenge. To this end, a recent post hoc analysis revealed that metformin had no effect on clinically relevant outcomes in patients with IPF. A plethora of posters also discussed the role of ECM in pulmonary fibrosis. Structure and composition of lung ECM had a notable role in fibroblast phenotype and cell differentiation, while normal matrix rigidity was protective against fibrosis and cancer. Classical ECM proteins including collagen and fibronectin, as well as proteins involved in signalling pathways such as epidermal growth factor, insulin-like growth factor and TCF- $\beta$ were differentially expressed between healthy, COPD and IPF matrix preparations. IPF myofibroblasts also exhibited higher activity of focal adhesion kinase (FAK) (phosphorylation of residue Y397) and protein kinase B (phosphorylation of S437) after TGF- $\beta$ stimulation than healthy controls. 
FAK was necessary for the aberrant collagen regulation induced by TGF- $\beta 1$. Another poster worth mentioning showed that galectin-3 was increased in bleomycin-treated mice and interestingly, galectin- 3 induced TGF- $\beta$ signalling in fibroblasts but not in epithelial cells. Finally, pathogenic commonalities between the fibrotic lung and the ageing lung were further enhanced by data showing that deletion of ETS domaincontaining protein Elk-1 gene (Elk1) resulted in age-related early fibrotic changes associated with the development of pulmonary fibrosis.

\section{Early career delegates session: maximising your publication output}

As mentioned above, one highlight of the LSC is always the dedicated Early Career Member session on Saturday afternoon. This year's session dealt with a vital aspect of every scientist's life: publications. The recently appointed chief editor of the European Respiratory Journal (ERJ), Martin Kolb (Hamilton, Canada) opened the session explaining the editorial procedures of the $E R J$, thus giving valuable information on what to expect when submitting papers to our society's main journal and how to write a manuscript [5]. Gisli Jenkins (Nottingham, UK), one of the editors in chief of Thorax focused on how the ever-stronger world of open access is impacting the culture of scientific publications and its possibilities, as well as many yet unaddressed caveats. Neil Bullen (Sheffield, UK), the managing editor of the $E R J$, demonstrated to the audience the different available metrics to track the impact of their publications on the scientific community [6]. Finally, we were happy to welcome Paul Noble (Los Angeles, (A, USA), who gave us his perspective, as a previous deputy editor of the Journal of Clinical Investigation, of writing articles for a general medical journal. In addition to the lectures by this expert panel, there was a lively discussion with members of the audience. Amongst other topics, we discussed the common fear that reviewers could bias the reviewing process itself, and on the impact and problems arising of pre-print platforms (e.g. arXiv.org). During the direct adjunct networking event, Early Career Members again had the opportunity to interact with our invited speakers and other members of the faculty, and further engage in discussions on this and other topics of interest.

\section{Evening pre-dinner talk}

The end of this Saturday full of science was a brilliant presentation by Peter Friedl (Nijmegen, The Netherlands) as a pre-dinner talk, who talked about visualisation of cell-matrix interactions during immune cell interactions and tumour invasion. Dr Friedl impressed the audience with stunning images and real-time videos of tumour invasion. Using intravital multiphoton microscopy, he showed how tumour microniches provide routes that promote cancer cell invasion along tracks that offer minimum resistance. He highlighted how plastic cancer cells are in their invasion patterns in response to tissue topology. Tumour cells prefer to follow a "collective invasion" model along vascular or neural vessels, which provide linear tracks for the tumour to migrate, in a fashion similar to highways. However, they adapt to a more "discontinuous invasion" pattern when tissues have a more complex structure, such as adipose tissue or renal glomeruli, and totally discontinuous in tissues that offer a mechanical challenge, such as connective tissue, where tumour cells are forced to migrate isolated. Through three-dimensional ultrastructure analyses, he showed the pre-defined geometry of the tracks. Additionally, he observed that tumour cells follow a "cell jamming" mechanism, without visible ECM degradation by proteases, but managing to push the surrounding tissues to make room for the advance front, where $\beta 1 / \beta 3$ integrins play an essential role.

\section{Reconstructing the matrix: bioengineering approaches}

The last day of the LSC was opened by a talk entitled "Lung Bioprinting: opportunities and challenges" by Ramon Farré (Barcelona, Spain). During the presentation, Dr Farré showed the opportunities and challenges of lung bioprinting and explained the different available techniques (extrusion, droplet and laser-based bioprinting). In addition, he explained the current situation in this field, where the reconstruction of a whole lung is still far-fetched but the available knowledge will allow us to perform new and more realistic in vitro disease models and drug testing in a short period of time. However, the use of these technologies still has some issues that need to be addressed. In this regard, improvements in substrate stiffness/ composition, oxygen and carbon dioxide diffusion, cyclic stretch, and shear stress still need to be introduced in the future models. The second talk of this session was given by Darcy Wagner (Lund, Sweden), who talked about ex vivo bioengineering approaches to develop human lung scaffolds. One way to obtain lung scaffolds is via decellularisation of native lungs, i.e. removal of all cells, while retaining the architecture of the lungs as well as ECM composition. She showed that decellularised lungs from healthy or diseased patients retain distinct protein profiles and that this pattern holds even when deep proteomic approaches are used. For instance, the heterogeneity of IPF tissue and the short-term viability in emphysematous lungs increase the difficulty of the decellularisation process. This indicates that each scaffold derived from human patients will be different. In parallel, this technology can open new doors for studying cell-ECM interactions and provide new insights into chronic lung disease pathomechanisms. 


\section{Drugging the matrix}

The last session of this year's LSC dealt with approaches to drug a diseased or altered matrix. In the first presentation of the session, Gisli Jenkins talked about targeting and profiling ECM components in IPF patients. He summarised that $\mathrm{G}$-protein coupled receptor (GPCR) signalling and mechanotransduction pathways involving RhoA are essential for pathogenesis of pulmonary fibrosis. He showed that injury to the alveolar epithelium results in mechanotransduction signals leading to $\alpha v \beta 6$ integrin-mediated activation TGF- $\beta$ via RhoA, resulting in fibrogenesis. However, in lung fibroblasts, mechanotransduction pathways involving cyclical mechanical stretch promotes TGF- $\beta$ secretion and can be reduced in Gaq/11-null mouse embryonic fibroblasts. In short, he presented that the effects of GPCR signalling in epithelial cells and fibroblasts are cell specific and may echo distinct endotypes of pulmonary fibrosis that might be targeted specifically for fibrosis therapy. Morten Karsdal (Herlev, Denmark) continued the session with his talk about the neoepitopes of ECM molecules for use as biomarkers. The relative amounts of interstitial matrix versus basement membrane collagens alter greatly throughout fibrosis in their amounts, locations and most importantly, the products of their proteolytic degradation. He emphasised the collagens' structure and function in matrices of IPF and COPD, and gave examples for recently discovered signalling functions of a few collagens: fragments of type IV collagen (tumstatin), type IV collagen (endotrophin), type VIII collagen (vastatin), type XV (restin) and type XVII collagen (endostatin). Dr Karsdal concluded that the biomarkers of the ECM can both hold prognostic value for disease status and help better understand disease progression.

\section{Insights into cell-matrix interactions in lung development}

Since 2017, there has been a group within ERS Assembly 7 (Group 7.08) focusing on lung and airway developmental biology. This new group within the Paediatrics Assembly focuses on lung and airway development, and its relationship with respiratory health during childhood and beyond. This group also aims to address the early (paediatric) origins of adult lung disease and the long-term sequelae of early lung disease. While the majority of presentations at the LSC focused on cell-matrix interactions in adult disease, there were a number of oral and poster presentations that focused on lung development and neonatal lung disease and were of special interest for Group 7.08 members. Marko Nikolic (Cambridge, UK), who received the award for the best oral presentation, shared intriguing data on his in vitro organoid culture system for human embryonic lung epithelial stem cells to recapitulate lung development [4]. Taking a side-step to dermal wound repair, Yuval Rinkevich (Munich, Germany) presented data on the existence of a scar-promoting fibroblast subtype identified by Engrailed 1 and CD26/dipeptidyl peptidase-4, which only emerges in late fetal development [7]. During the Young Investigator Session, Jennifer Collins showed how $\mathrm{CD}_{146^{+}}$mesenchymal stromal cells isolated from hyperoxia-injured neonatal rat lungs have impaired angiogenic supportive capacity and an altered gene expression profile. In addition, there were a number of poster presentations that focused on neonatal lung disease and lung development by Maeva Zysman (Vincennes, France), Koni Ivanova (Stara Zagora, Bulgaria), Sander van Riet (Leiden, the Netherlands) and Anne Hilgendorff (Munich, Germany). Taken together, LSC 2018 was a promising first event for Group 7.08, providing a fertile ground for inspiration for future research.

\section{Conclusion}

This year's LSC has once again been a brilliant meeting, bringing together leading experts in the field to discuss recent scientific findings. The relatively small setting provides excellent means for networking and the establishment of future collaborations. Early Career Members can only be encouraged to attend this fantastic conference in the future. Join us next year; the topic will be "Mechanisms of acute exacerbations in respiratory disease" and the scientific programme will be outstanding as always.

\footnotetext{
Acknowledgements

The authors' affiliations are as follows. I. Almendros: Unitat de Biofísica i Bioenginyeria, Facultat de Medicina i Ciències de la Salut, Universitat de Barcelona, Barcelona, and Centro de Investigación Biomédica en Red de Enfermedades Respiratorias, Madrid, Spain; H.N. Alsafadi: Dept of Experimental Medical Science, Lung Bioengineering and Regeneration, and Wallenberg Center for Molecular Medicine, Lund University, Lund, Sweden; D. Bölükbas: Dept of Experimental Medical Science, Lung Bioengineering and Regeneration, and Wallenberg Center for Molecular Medicine, Lund University, Lund, Sweden; J.J.P. Collins: Dept of Pediatric Surgery, Erasmus University Medical Centre, Rotterdam, the Netherlands; P. Duch: Unitat de Biofísica i Bioenginyeria, Facultat de Medicina i Ciències de la Salut, Universitat de Barcelona, Barcelona, and Centro de Investigación Biomédica en Red de Enfermedades
} 
Respiratorias, Madrid, Spain; E.M. Garrido-Martin: H120-CNIO Lung Cancer Clinical Research Unit, Research Institute Hospital 12 Octubre - Spanish National Cancer Research Centre, and Biomedical Research Networking Centre Consortium of Cancer, Madrid, Spain; N. Kahn: Pneumology and Critical Care Medicine, Thoraxklinik at Heidelberg University Hospital, and Translational Lung Research Center, Member of the German Center for Lung Research (DZL), Heidelberg, Germany; T. Karampitsakos: 5th Dept of Pneumonology, Hospital for Thoracic Diseases, "Sotiria", Athens, Greece; I. Mahmutovic Persson: Institution of Medical Radiation Physics, Lund University, Dept of Translational Medicine, Malmö, Sweden; A. Tzouvelekis: 1st Academic Dept of Pneumonology, Hospital for Thoracic Diseases, "Sotiria", Medical School, National and Kapodistrian University of Athens, and Division of Immunology, Biomedical Sciences Research Center "Alexander Fleming”, Athens, Greece; F.E. Uhl: University of Vermont, College of Medicine, Burlington, VT, USA; S. Bartel: Early Life Origins of Chronic Lung Disease, Research Center Borstel, Leibniz Lung Center, Member of the DZL, Borstel, Germany.

\section{Conflict of interest}

S. Bartel has received personal fees from Bencard Allergie $\mathrm{GmbH}$ for serving as a member of an advisory board and a project grant from Bencard Allergie $\mathrm{GmbH}$, outside the submitted work.

\section{References}

1. Papakonstantinou E, Roth $\mathrm{M}$, Klagas I, et al. COPD exacerbations are associated with proinflammatory degradation of hyaluronic acid. Chest 2015; 148: 1497-1507.

2. Burgstaller G, Oehrle B, Gerckens $M$, et al. The instructive extracellular matrix of the lung: basic composition and alterations in chronic lung disease. Eur RespirJ 2017; 50: 1601805

3. Hinz B, Suki B. Does breathing amplify fibrosis? Am J Respir Crit Care Med 2016; 194: 9-11.

4. Nikolic MZ, Caritg O, Jeng Q, et al. Human embryonic lung epithelial tips are multipotent progenitors that can be expanded in vitro as long-term self-renewing organoids. Elife 2017; 6: e26575.

5. Sterk PJ, Rabe KF. The joy of writing a paper. Breathe 2008; 4: 224-232.

6. Smith R. Alternative metrics for measuring the quality of articles and journals. Ecancermedicalscience 2013; 7: ed18.

7. Rinkevich Y, Walmsley GG, Hu MS, et al. Skin fibrosis. Identification and isolation of a dermal lineage with intrinsic fibrogenic potential. Science 2015; 348: aaa2151. 